\title{
Investigation on Self-Care Ability and Morning Running Training Management of Rural Boarding Left Behind Children
}

\author{
Zhang Yuanping \\ School of Art and Design, Heyuan Polytechnic, Heyuan, Guangdong, China \\ zypball0603@126.com
}

\begin{abstract}
With the acceleration of China's modernization process, family based migration based on husband and daughter has made the upbringing and education of migrant workers' children a social problem. Due to policy and many practical problems, most migrant workers are separated from their children. These left behind children are either taken care of by a single parent at home or by someone other than their parents. Most of the left behind children can only stay in boarding schools for long-term management and learning, which is the "problem of left behind children" widely concerned by the society. The education problem of left behind children is not obvious in primary school. After entering the junior middle school, the related problems and performance of left behind children education are very prominent. The dropout rate of left behind children in junior middle school increases obviously with the grade. Therefore, it is a very urgent task to consolidate and strengthen the junior middle school education of left behind children. Based on the data analysis of 150 valid questionnaires, it is concluded that the intervention factors for the learning enthusiasm of rural residential left behind children are reasonable and appropriate collective activities and appropriate education and teaching guidance Guide. The main factors that affect their learning energy, self-care ability and self-discipline are energy and lack of good work and rest rules, which leads to the vicious circle of not concentrating in class and not having a good rest at night. Therefore, as a morning run that can play an exercise role and is also a collective activity, it has become a relatively excellent sports to improve the self-care ability and even learning enthusiasm of rural residential left behind children. It can be clearly pointed out that the training and management of morning running is suitable to join the boarding management system of the left behind children in rural boarding system. Boarding schools should enhance the students' sense of ownership in education, help them to improve their ability of independent learning and self-care, and also appropriately increase other student activities. Left behind children are often lack of collective care. They are properly given the opportunity to communicate and express in the collective, communicate with each other, form and develop their social adaptability, so as to achieve the purpose and effect of education.
\end{abstract}

Keywords: left behind children, boarding, self-care ability, morning running

\section{农村寄宿制留守儿童自理能力调查与晨跑训练管理}

\author{
张原平
}

河源职业技术学院艺术与设计学院, 河源, 广东, 中国

zypball0603@126.com

\begin{abstract}
摘要
随着中国现代化进程的加快，以家庭为单位的以丈夫和女儿为单位的移徙已使移徙工人子女的抚养和 教育成为一个社会性问题，由于政策和许多实际问题，大多数移民工人与子女分离。这些留守儿童要 么由家里的单亲父母照顾, 要么由父母以外的其他人照顾。大多数留守儿童只能留在寄宿学校进行长 期管理和学习, 这是社会广泛关注的 “留守儿童问题”。留守儿童的教育问题在小学阶段并不明显, 步入初中阶段后, 留守儿童教育的相关问题以及表现就很突出。初中阶段留守儿童的辍学率随年级而 增长明显。因此, 巩固和加强留守儿童的初中教育是一项非常紧迫的任务。通过对 12 个初中农村寄宿 制留守儿童自理能力的问题进行相关调查分析, 其中 5 道为自律性选择题目, 7 道为自理能力调查选择
\end{abstract}


题目; 基于150份有效问卷进行数据分析, 得出农村寄宿制留守儿童学习积极性的可干预因素在于进行 合理合适的集体活动和适当的教育教学引导。影响他们学习精力与自理能力和自律性的因素主要是精 力旺盛与缺乏良好的作息规律，从而导致课堂精力不集中和夜晚无法良好休息的恶性循环。因此作为 可以起到锻炼作用及同时也是集体活动的晨跑，就成为较为优秀的提升农村寄宿制留守儿童自理能力 甚至学习积极性的体育运动。可以明确指出的是, 晨跑的训练与管理适合加入农村寄宿制留守儿童的 寄宿管理制度当中。寄宿制学校在教育方面要增强学生的主人翁精神, 帮助他们提升自主学习和自理 的能力, 也可以适当增加其他学生活动。留守儿童往往更缺乏集体关怀, 适当的给予他们在集体的交 往和表达的机会, 相互沟通并形成和发展其社会适应能力, 从而达成教育的目的与效果。

关键词: 留守儿童, 寄宿, 自理能力, 晨跑

\section{1. 研究目的}

自 1980 年代初以来，中国农村剩余劳动力不断向 着城市转移。农村青壮劳动人口向城市的迁移经历了三 个阶段：第三阶段是 1995 年左右。尽管目前移徙农民 的总数并没有迅速增长, 但以家庭为单位的以丈夫和女 儿为单位的移徙已使移徙工人子女的抚养和教育成为 一个社会性问题。由于政策和许多实际问题, 大多数移 民工人与子女分离。这些留守儿童要么由家里的单亲父 母照顾, 要么由父母以外的其他人照顾, 例如祖父母, 兄弟姐妹, 亲戚, 邻居等。大多数留守儿童只能留在寄 宿学校进行长期管理和学习。这是社会广泛关注的 “留 守儿童问题” [1]。

从教育者的角度来看, 留守儿童的问题是教育问 题。在研究留守儿童在农村寄宿。大多数研究都得出了 类似的结论: “与非留守儿童相比, 留守儿童更容易具 有不良学习习惯” ${ }^{[2]}$ 。有研究认为, 留守儿童的教育问 题在小学阶段并不明显, 步入初中阶段后, 留守儿童教 育的相关问题以及表现就很突出。初中阶段留守儿童的 辍学率随年级而增长明显。留守儿童的初中教育是对于 留守儿童的教育与未来发展都十分关键。在行为和交流 方面, 一些研究表明, “外出务工父母对初中生的社交 行为和社交技能有负面影响” ${ }^{[3]}$ 。

而如何通过学校教育对父母外出务工的留守儿童 进行良好的教导, 应该引起有关部门的关注, 并引起对 留守儿童问题的关注。晨跑是一种主要早晨进行的运 动。坚持晨跑可以增强身体健康, 提高免疫力并改善精 神状态。“晨跑前后, 应进行一些热身伸展运动, 以减 少身体状况不利的可能性” ${ }^{[4]}$ 。晨跑不仅是一项体育运 动, 而且是一项集体活动, 可能对农村寄宿制留守儿童 的自我保健和学习热情产生影响。从教育的角度来看, 本研究期主要是调查并研究学生的自理能力, 分析问题 并寻找与晨跑训练管理相结合的寄宿学生管理方式, 以 帮助提高学生的自律性和学习积极性。

\section{2. 研究方法}

本研究通过定性与定量研究结合法, 采用文献法、 问卷调查法、定点访谈法等收集资料, 并作为文章主要 依据，对文章核心问题进行探讨。

\section{1. 文献法}

文献法在选题、基础资料搜集及作为依据支持研究 方面意义重大。通过文献阅读与搜集把握农村留守儿童 自理能力与学校寄宿情况, 明确概念和理论, 确定文章 要点与创新点, 深化对文章选题及核心研究关键点的认 识和理解, 并把握现有研究的不足和缺陷, 以此提出本 研究的突破与创新。

\section{2. 问卷调查法}

在参考相关文献及研究的现状后, 围绕研究核心设 计农村寄宿制学校留守儿童自理能力问卷, 以了解农村 寄宿制学校留守儿童的自理能力和教育状况。

\section{3. 定点访谈法}

访谈均采取定点访谈, 对象为农村中学教师, 以及 部分留守儿章。访谈时间及地点由调查者和被访谈者商 量决定, 一般是在被访谈者工作或学习的场所。访谈力 求真实客观, 以帮助后期对问题的分析和解决。

\section{4. 核心界定}

\subsection{1. 留守儿童}

有研究认为, 高山留守儿童的心理状况好于初中留 守儿童, 年龄越小心理问题越突出。而初山义务教育也 是农村留守儿童父母选择打工的一个关键时期, 留守儿 童问题表现的比较突出。因此, 本研究把 “留守儿童” 定义为, “由于父母双方或一方外出打工且外山打工年 限在一年以上, 而被留在农村的家乡, 并且需要其他亲 人或委托人照顾的处于义务教育阶段的初中留守寄宿 的儿童” ${ }^{[5]}$ 。 


\subsection{2. 寄宿制及寄宿制学校}

寄宿制是指学校有向学生提供住宿条件的能力, 而 不是作为一种学生必须接受的学校制度, 强制要求学生 住宿, 学生可以自由选择是否住宿。本文将寄宿制学校 界定为具有可以满足学生基本生活需要的宿舍、食堂以 及其他相关生活设施的学校。

\section{5. 自理能力}

本次研究将自律性与自理能力分为两个模块, 自律 性为必要生活活动外的软指标 (即无硬性要求的指标), 自理能力为必要生活活动硬指标 (即需要明确是否完成 的指标）。

\subsection{1. 自律性}

量化指标: 此部分问卷设置 5 道调查问卷题目, 分 别为: 1. 寄宿期间每天自主学习时间 ( A: 1 小时, B: 2 小时, C: 3 小时以上）；2. 衣物换洗间隔时间（A: 1 天， $\mathrm{B}: 2^{\sim} 3$ 天， $\mathrm{C}: 3$ 天以上）；3. 每天自主体育活动时 间 (A: 半小时, B: 1 小时, $C: 2$ 小时以上)； 4 . 对自 主学习与自主体育互动的自主选择 $(\mathrm{A}$ : 偏好自主体育活 动， $\mathrm{B}$ : 偏好自主学习, $\mathrm{C}$ : 无特别偏好)；5.上课时间 分心原因 ( $\mathrm{A}$ : 希望外出活动, $\mathrm{B}$ : 难以理解课程内容, $C$ ：疲急或劳累，其他：自填）。

\subsection{2. 自理能力}

量化指标: 此部分问卷共 7 道选择题, 设置其中 5 道题目, 答案设置仅为是或否, 是为 $\mathrm{A}$, 否为 $\mathrm{B}$, 分别 为: 6 . 能否按时起床; 7 . 能否按时进入教室上课; 8 . 能否按宿舍管理要求处理内务; 9. 能否按时熄灯睡觉, 10. 能否打理好个人卫生。其余两道题目为: 11 . 有无法 良好休息时无法良好休息的原因（A 无此情况， B 学习 劳累, C 身体疲㤂, D 精力过剩)；12. 倾向于何种锻炼 与运动 $(\mathrm{A}$ 跑步, $\mathrm{B}$ 球类, $\mathrm{C}$ 热身舒展类, $\mathrm{D}$ : 球拍类)

\section{3. 结果与分析}

本次研究通过调查问卷的填写及对个别学生及教 师的访谈进行分析, 通过县城乡镇中学教师下放问卷共 200 份, 最终收回 167 份, 剔除填写不全或随意涂抹的 问卷后有效问卷共 150 份。对 1 名县城乡镇中学教师进 行访谈和对 2 名知情同意的学生进行沟通访谈。分析结 果如下:

根据图 1 可知大部分农村寄宿制留守学生学习时 间少于 2 小时, 只有不到五分之一的学生每天自主学习 3 小时以上。衣物换洗时间大部分为 $2^{\sim} 3$ 天换洗一次, 但是 150 人中依旧有 51 人, 超过三分之一换洗衣物时
间超过 3 天。自主体育活动时间只有 20 人活动在半小 时左右, 其他都在 1 小时以上。大部分学生选择喜欢体 育活动而非学习, 一部分选择无偏好, 只有 19 人选择 喜好自主学习。而图 2 大部分学生缺乏学习积极性的原 因是希望外出活动, 在其它的原因里, 其中自填部分有 7 人提到感觉自身比较好动, 学校体育活动较少, 感觉 在课堂内难以安坐。

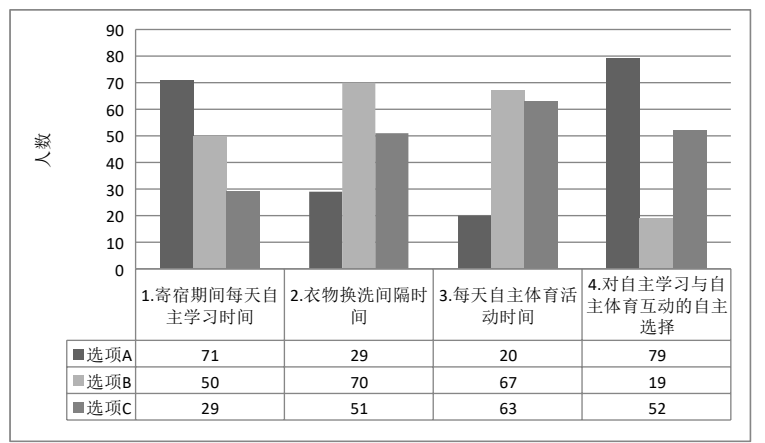

图 1（寄宿学生学习时间调查分布图）

图 1 为 1 到 4 题选择题调查的具体情况: 1 . 寄宿期 间每天自主学习时间 (A: 1 小时, B: 2 小时, C: 3 小 时以上)；2. 衣物换洗间隔时间 ( $A: 1$ 天, $B: 2^{\sim} 3$ 天, $C$ : 3 天以上）；3. 每天自主体育活动时间 ( $\mathrm{A}$ : 半小时, $\mathrm{B}$ : 1 小时, $C: 2$ 小时以上)； 4 . 对自主学习与自主体育互 动的自主选择 ( $\mathrm{A}$ : 偏好自主体育活动, $\mathrm{B}$ : 偏好自主学 习, C：无特别偏好）。

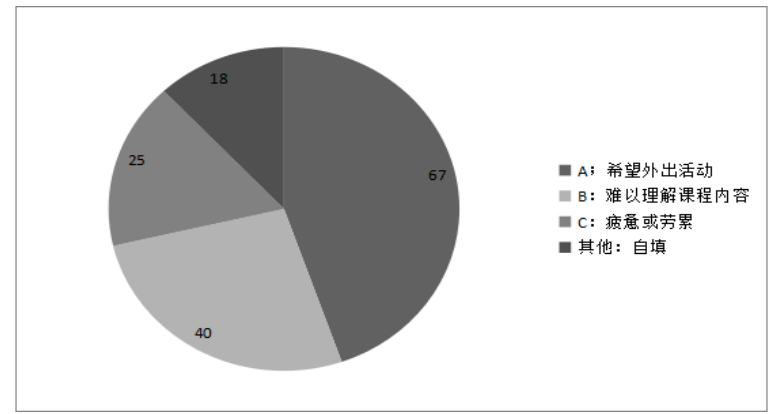

图 2（学生课程分心原因统计表）

图 2 为调查题目 5 上课时间分心原因具体情况 (A: 希望外出活动, $\mathrm{B}$ : 难以理解课程内容, $\mathrm{C}$ : 疲备或劳累, 其他: 自填)。

在图 3 中大部分学生是可以按照要求完成相关作 息和学校规定的, 值得注意的是通过 6 和 7 两道题对比, 很多人无法按时起床, 但是能够按时上课的人数比能够 按时起床的人数多, 表明存在赖床的情况。而通过问题 9 可知能按时熄灯睡觉的人数较少, 这也印证了 6 和 7 两个问题的情况。根据图 4 可知, 无法良好休息的原因 很大程度上是精力过剩, 而 12 题显示, 学生最希望的 运动跑步最佳, 其次是热身舒展类, 然后才是球类与球 拍类。 


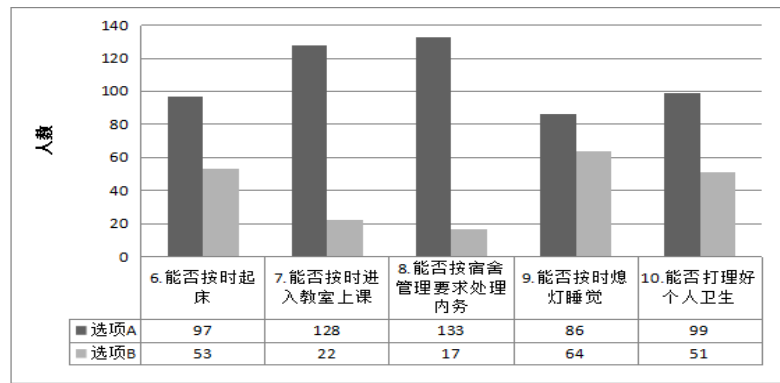

图 3（学生作息规律调查分布图）

图 3 为 6 到 10 题的具体情况, 答案设置仅为是或 否, 是为 $A$, 否为 $B$, 分别为: 6 . 能否按时起床; 7 . 能 否按时进入教室上课; 8. 能否按宿舍管理要求处理内 务；9. 能否按时熄灯睡觉，10. 能否打理好个人卫生。

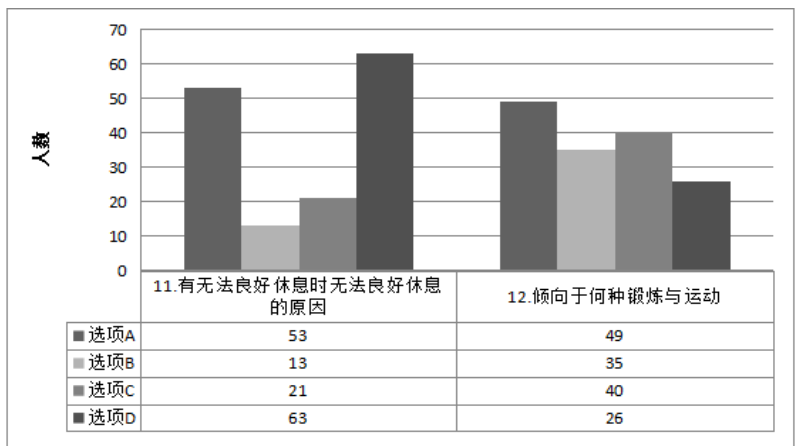

图 4（学生休息状态与喜爱运动项目调查分布图）

图 4 为第 11 与 12 题的具体情况: 11 . 有无法良好 休息时无法良好休息的原因 (A 无此情况, B 学习劳累, C 身体疲畕, D 精力过剩)；12. 倾向于何种锻炼与运动 ( $\mathrm{A}$ 跑步, B 球类, C 热身舒展类, D 球拍类)

通过访谈可知, 留守儿童普遍存在学习或者活动的 积极性不足和平时的情绪激烈的问题, 而通过两个学生 的访谈印证, 发现积极性和情绪问题大多在于家长的外 出务工, 而让他们无法积极参与学习的因素, 也有他们 缺乏合适的活动和同学一起融洽参与。

通过以上的结果, 我们可以初步得出结论, 影响农 村寄宿制留守儿童学习积极性的可干预因素在于合理 合适的活动和适当的引导, 让他们融入学习与生活。同 时, 影响他们学习精力与自理能力和自律性的因素主要 是精力旺盛, 无法按时休息, 导致的无法按时起床, 导 致课堂精力不集中和夜晚无法良好休息的恶性循环。因 此, 对于该类现象, 本研究认为通过合理合适的体育锻 炼来平衡学生学习和运动的欲望最为合适, 而且跑步和 热身舒展运动在本次调查中选择人数居高, 可以二者协 同进行, 晨跑可以加强学生体质, 更能促进学生的良好 作息, 减少学生的活动欲望, 使学生更加专注于课堂和 自主学习。

\section{4. 结论与建议}

通过对 12 个农村寄宿制留守儿童自理能力的相关 调查分析, 得出农村寄宿制留守儿童学习积极性的可干 预因素在于合理合适的活动和适当的引导, 影响他们学 习精力与自理能力和自律性的因素主要是精力旺盛, 无 法按时休息, 导致的无法按时起床, 导致课堂精力不集 中和夜晚无法良好休息的恶性循环。因此作为可以起到 锻炼作用及同时也是集体活动的晨跑, 就成为较为优秀 的提升农村寄宿制留守儿童自理能力甚至学习积极性 的体育运动。同时晨跑可以配合热身舒展类运动, 因此 晨跑运动据此次研究结果来看完全适合农村寄宿制留 守儿童的学习与生活的自律性提升。

有学者指出“教育要为学生的幸福人生奠定一个坚 实的基础。因此, 我们特别需要幸福教育”。重要的不 是让学生成为某领域的人才, 而是要让他们成为积极的 生活者。“学校非常首要的职责就是帮助他们确立健康 的、文明的生活观念和态度” ${ }^{[6,7]}$ 。要增强学生的主人 翁精神, 教育的最终目标是让学生学会自我教育。“寄 宿制学校还可以充分利用其师资、场地和设施的优势, 开展名目繁多、内容丰富的课外活动和社团活动, 以及 开展春游、秋游、野营之类的旅游活动让学生在社会这 个大环境中感受和体验, 吸引学生” ${ }^{[8]}$ 。“留守儿童更 需要集体的帮助, 在集体的交往中进行自我表达, 相互 沟通相互合作, 并以此形成和发展着自己的社会能力”。 “学生良好的表现所形成的氛围, 也是一种教育资源” [9-11]。

\section{REFERENCES}

[1] Ye J., Murray J. (2005) Focus on left behind children. Book excerpt,12: 14-15.

[2] Gao H. (2009) A review of the research on the education of left behind children. Journal of Hebei Normal University (EDUCATION SCIENCE EDITION), 04: 27-31.

[3] Yang M., Gong W., Wang J. (2015) Research on education of left behind children in rural areas. The first epoch star Innovation Education Forum

[4] Meng J. (2018) Pay attention to scientific outdoor morning running. Healthy life, 9 .

[5] Wang Y. (2011) Comparative study on mental health of left behind children in rural junior middle schools [J]. Journal of University of Electronic Science and Technology (SOCIAL SCIENCE EDITION), 13 (3): 97-101.

[6] Ma Q. (2019) Views on education of left behind children in rural areas. Good parents, 6: 215-215.

[7] Xu Q. (2007) Paying attention to the education of left 
behind children and building a harmonious new countryside $[\mathrm{J}]$. Modern agricultural science and technology, 011: 156-157.

[8] Hu Y. (2019) Education problems of rural left behind children and effective countermeasures. Teachers, 7: 24-25.

[9] Zhang L. (2019) On the position and role of students' self-management in the management of College Students' Education . Science public (Science Education), 3.

[10] Zhang G. (2019) Education students in mind. Exam weekly, 32: 49-49.

[11] Gao R. (2019) On the impact of labor education on the mental health of primary school students. 